\title{
Astragaloside IV Inhibits Mitochondrial-Dependent Apoptosis of the Dorsal Root Ganglion in Diabetic Peripheral Neuropathy Rats Through Modulation of the SIRTI/p53 Signaling Pathway
}

This article was published in the following Dove Press journal:

Diabetes, Metabolic Syndrome and Obesity: Targets and Therapy

\author{
Ying Ben $\mathbb{D}^{1,2, *}$ \\ Juan $\mathrm{Hao}^{\mathrm{l}, *}$ \\ Zhihong Zhang $\mathbb{D}^{1,2}$ \\ Yunzhao Xiong' \\ Cuijuan Zhang $\mathbb{D}^{1,2}$ \\ Yi Chang ${ }^{1,2}$ \\ Fan Yang ${ }^{1,2}$ \\ Hui Li ${ }^{1,2}$ \\ Tianya Zhang' \\ Xiangting Wang ${ }^{1,2}$ \\ Qingyou $X u^{1,2}$
}

'Hebei University of Chinese Medicine, Shijiazhuang, Hebei, People's Republic of China; ${ }^{2}$ Hebei Key Laboratory of Integrative Medicine on Liver-Kidney Patterns, Hebei University of Chinese Medicine, Shijiazhuang, Hebei, People's Republic of China

*These authors contributed equally to this work
Correspondence: Qingyou Xu

Hebei University of Chinese Medicine, No. 326 Xinshinan Road, Qiaoxi District, Shijiazhuang, Hebei Province, 050090

People's Republic of China

Tel +86 I3832368865

Fax +8631189926000

Email qingyouxu@sohu.com
Purpose: To investigate the effect of astragaloside IV (AS-IV) on mitochondrial-dependent apoptosis in the dorsal root ganglion of diabetic peripheral neuropathy (DPN) rats through the SIRT1/p53 pathway.

Methods: Diabetic rat model was induced by high-carbohydrate/high-fat diet and intraperitoneal injection of STZ. Diabetic rats were divided into three groups ( $n=16$ per group): DPN group, AS-IV group $(60 \mathrm{mg} / \mathrm{kg} / \mathrm{d})$ and $\alpha$-lipoic acid (ALA) group $(60 \mathrm{mg} / \mathrm{kg} / \mathrm{d})$. Weight and blood glucose levels were monitored every 4 weeks for 12 weeks. DPN was evaluated using the Von Frey Filaments Test and nerve conduction velocity. The dorsal root ganglia of rats were isolated and the pathological changes of mitochondria were observed by electron microscopy. The activity of mitochondrial electron transport chain complex, mitochondrial membrane potential, malonaldehyde (MDA) and glutathione (GSH) levels were measured. Neural apoptosis was detected using the Terminal Deoxynucleotidyl Nick-End Labeling (TUNEL) assay kit. The cleaved caspase-3, major proteins in the SIRT1/p53 pathway, including SIRT1, acetyl p53, Drp1, BAX, and BCL-2, were detected using immunohistochemistry and Western blot. Gene expression of major proteins in the SIRT1/ p53 pathway was also detected.

Results: After 12 weeks of treatment, AS-IV and ALA did not significantly affect body weight or fasting glucose levels, but reduced mechanical abnormal pain in DPN and improved nerve conduction velocity. AS-IV and ALA increased the level of GSH and decreased the level of MDA. Both AS-IV and ALA can reduce mitochondrial damage, improve mitochondrial electron transport chain complex activity and mitochondrial membrane potential, and reduce the percentages of positive cells with DNA fragmentation and the expression of cleaved caspase-3 protein. AS-IV and ALA up-regulated the expression of SIRT1 and down-regulated the expression of acetyl-p53, Drp1 and the ratio of BAX to BCL-2. Changes in gene expression were similar.

Conclusion: AS-IV can reduce the occurrence of mitochondrial-dependent apoptosis by regulating the SIRT1/p53 pathway. It has a similar therapeutic effect as ALA and is therefore a promising drug for the potential treatment of DPN.

Keywords: astragaloside IV, diabetic peripheral neuropathy, dorsal root ganglion, mitochondria, apoptosis, SIRT1/p53 pathway

\section{Introduction}

Diabetic peripheral neuropathy (DPN) is one of the most common chronic complications in diabetic patients, and its prevalence can reach $50 \%$. ${ }^{1}$ This condition is characterized by pain, paresthesia, and sensory loss. In severe cases, refractory ulcers may appear, which 
may eventually lead to amputation of the affected limb. Unfortunately, successful treatment and prevention remain challenging due to a lack of knowledge regarding the mechanisms of DPN development. ${ }^{2}$ Studies have shown that strict glycemic control ameliorates but does not reverse neuropathy in type 1 patients. ${ }^{3,4}$ By contrast, glycemic control is relatively ineffective in preventing diabetic neuropathy in type 2 diabetes. $^{5}$

Mitochondrial damage in dorsal root ganglion (DRG) neurons, axons and Schwann cells has been proposed as a unifying mechanism for diabetic neuropathy. ${ }^{6-10}$ Studies have shown that $\mathrm{p} 53$ promotes apoptosis by regulating mitochondrial fission. ${ }^{11}$ p53 can be transported from the nucleus to the cytoplasm, where it assists in translocating Drp1 to the mitochondrial surface, ${ }^{12}$ causing the mitochondria to split into several fragments and affecting its function. ${ }^{13}$ At the same time, p53 can upregulate BAX expression downregulate BCL-2 expression, increase the permeability of the mitochondrial outer membrane, and induce cytochrome $\mathrm{C}$ release from the mitochondrial intermembrane space to initiate apoptosis.

Sirtuin 1 (SIRT1) is a protein deacetylase that depends on nicotinamide adenine dinucleotide (NAD+). SIRT1 catalyzes the deacetylation of $\mathrm{p} 53$ at K382. ${ }^{14,15}$ Based on recent studies, the generally held view is that the function of p53 is inhibited by deacetylation of $\mathrm{K} 382$ at the C-terminus. ${ }^{16,17}$ The inhibition of $\mathrm{p} 53$ function may be a major mechanism for the anti-aging and cell survivalpromoting function of SIRT1. ${ }^{8,18-20}$

Astragaloside IV (AS-IV) is a biologically active saponin isolated from Astragalus root. Some studies on neurodegenerative diseases (such as Parkinson's disease and Alzheimer's disease) have shown that AS-IV has a protective effect on mitochondrial damage and can inhibit neuronal apoptosis. $^{21-23}$ In addition, AS-IV also has a strong antioxidative stress effect. ${ }^{24,25} \alpha$-Lipoic acid (ALA) is a wellknown antioxidant and has been widely used to treat DPN. ${ }^{26,27}$ Studies have found that it can up-regulate SIRT1 expression. $^{28,29}$ Based on the above beneficial effects, we speculate that AS-IV can protect mitochondria and inhibit the apoptosis of dorsal root ganglion neurons through the SIRT1/ p53 pathway. Therefore, in this experiment, we used ALA as a positive control to study the beneficial effects of AS-IV on the dorsal root ganglia of diabetic rats induced by streptozotocin adjuvant and a high-carbohydrate/high-fat diet. Our research aimed to provide some clues to prove that AS-IV may be an effective and feasible drug candidate for the treatment of diabetic peripheral neuropathy.

\section{Materials and Methods}

\section{Animals}

Sixty-four male Sprague Dawley rats (3 weeks old, $110 \pm 10 \mathrm{~g}$ ) were purchased from Vital Rive Experimental Animal Technology Co., Ltd. (Beijing, China) SCXK [Beijing] 20160006. The animals were placed in a $12 \mathrm{~h} / 12 \mathrm{~h}$ artificial lightdark cycle environment with a temperature of $22-24{ }^{\circ} \mathrm{C}$ and a humidity of $40 \%$. The animals were kept in 16 cages with 4 rats in each cage, and the bedding was changed daily. All of the following experiments complied with the "Guiding Principles for the Care and Use of Laboratory Animals" promulgated by the National Science and Technology Commission of China.

\section{Induction of Diabetes and Drug Treatment}

Sixteen rats were randomly selected as the control group and given a standard diet. The remaining 48 rats were fed a highcarbohydrate/high-fat diet (67\% standard diet, 10\% lard, 20\% sucrose, $2.5 \%$ cholesterol, and $0.5 \%$ sodium cholate) for 6 weeks and were then intraperitoneally injected with streptozotocin (STZ) (Sigma-Aldrich, St Louis, MO, USA) (25 mg/kg) twice with an interval of 2 days. One week after the injection of STZ, animals with fasting blood glucose levels of $\geq 16.7 \mathrm{mmol} /$ $\mathrm{L}$ were used as diabetic animals and continued to be fed a highcarbohydrate/high-fat diet. Forty-eight diabetic rats were randomly divided into the DPN group, DPN plus ALA group, and DPN plus AS-IV group, with 16 rats in each group. The DPN plus ALA group was given $60 \mathrm{mg} / \mathrm{kg}$ of ALA (Sigma-Aldrich, St Louis, MO, USA) by intragastric administration daily, while the DPN plus AS-IV group was given $60 \mathrm{mg} / \mathrm{kg}$ of AS-IV (Chengdu Herbpurify Co., Ltd. Chengdu, China) daily; the control group and DPN group received the same amount of normal saline by intragastric administration every day. The drug treatment lasted 12 weeks.

\section{Fasting Blood Glucose and Weight}

The successful induction of diabetes was recorded as week 0 . The fasting blood glucose and body weight of the rats in each group were measured at the 4th, 8th and 12th week, respectively.

\section{Mechanical Hyperalgesia}

As described in Ueda and Neyama, ${ }^{30}$ the electronic von Frey anesthesia instrument was used to detect the pain threshold of the experimental rats (type 2390, $90 \mathrm{~g}$ probe, $0.8 \mathrm{~mm}$ diameter; IITC Life Science Inc., Woodland Hills, CA, USA). The rigid tip was used to mechanically stimulated the middle of the 
plantar surface of the rat hind paw, and the pressure required to induce the withdrawal response was defined as the pain threshold, which was recorded in grams. The test was repeated once every 5 minutes, with five measurements for each animal, and the average value was calculated.

\section{Nerve Conduction Velocity (NCV)}

After the experimental rats were anesthetized with $3 \%$ by volume isoflurane, $1 \mathrm{e} 1.2 \%$ isoflurane was added to oxygen through a mask to maintain anesthesia, and the rats were fixed in the prone position. Stimulating and recording platinum wire electrodes were placed directly under the sciatic nerve trunk of right leg. A piece of skin between the biceps femoris and semitendinous muscles was incised and detached to expose sciatic nerve at two sites to attach electrodes. Stimulation electrode was placed at the sciatic nerve notch. Sciatic nerve was stimulated with single square waves pluses $(1.2 \mathrm{~V}$ in intensity, $1 \mathrm{~ms}$ in width) through the Functional Experiment System (BL-420s, Taimeng, Sichuan, China). Action potential latency (L) of sciatic nerve and the distance (D) between the two electrodes were measured to calculate the motor NCV $(\mathrm{MNCV}), \mathrm{MNCV}=\mathrm{D} / \mathrm{L}(\mathrm{m} / \mathrm{s})$. Sensory NCV (SNCV) was measured and recorded by exchanging the electrode of stimulation and that of recording. Stimulation parameters and SNCV calculation procedure were the same as MNCV methods.

\section{Histopathology and Immunohistochemistry}

Electron microscopy: The L4-5 dorsal root ganglia were fixed in $4 \%$ glutaraldehyde and treated with $1 \%$ OsO4 in $0.1 \mathrm{MPBS}$. The samples were dehydrated through successive application of graded acetone solutions and embedded in EPON 812. Ultrathin sections $(70 \mathrm{~nm})$ were placed on a copper grid and stained with uranyl acetate and lead citrate. The samples were observed by transmission electron microscopy (TEM, JEM1230, Tokyo, Japan). Pathological changes of mitochondria in neurons were assessed. The level of intact mitochondria was determined using the published Mitochondrial Quality Index (MQI). ${ }^{31}$ Briefly, individual mitochondria were assigned one of the following grades: 1 - fully intact, 2 - disruption of the cristae (typically from swelling or loss of integrity of the inner membrane), 3 - disruption of the outer mitochondrial membrane, and 4-disruption of the cristae and the outer membrane.

Immunohistochemistry: The L4-5 dorsal root ganglia were quickly collected and fixed in 4\% paraformaldehyde overnight. The tissue was then paraffinized and cut into $4 \mu \mathrm{m}$ sections. The sections were deparaffinized, the antigen was repaired with citric acid solution $(0.01 \mathrm{~mol} / \mathrm{L}, \mathrm{pH} 6.0)$, and the sections were then incubated with $3 \% \mathrm{H}_{2} \mathrm{O}_{2}$ to reduce nonspecific background staining caused by endogenous peroxidase. The sections were blocked with non-immunoreactive serum and then incubated with antibodies against SIRT1, acetyl-p53, Drp1, BAX, BCL-2, and cleaved-caspase- 3 at $4{ }^{\circ} \mathrm{C}$ overnight. The next day, the sections were incubated with goat anti-rabbit (Beijing noble Ryder Technology Co., Ltd., Beijing, China), stained with diaminobenzidine (DAB) chromogen, and then counterstained with hematoxylin. We observed and analyzed the sections using an optical microscope (Olympus, Bx53F, Tokyo, Japan). The primary antibodies were used in the following dilutions: SIRT1, 1:200 (Abcam, Cambridge, UK); acetyl-p53, 1:200 (Abcam, Cambridge, UK); Drp1, 1:400 (Abcam, Cambridge, UK); BAX, 1:400 (Protaintech, Rosemont, IL, USA); BCL-2, 1:200 (Affinity Bioscience, Cincinnati, OH, USA); and cleaved caspase-3, 1:200 (Cell Signaling, Danvers, MA, USA).

\section{Mitochondrial Function}

The mitochondria were extracted from the dorsal root ganglion according to the method of the Animal Tissue Active Mitochondria Isolation Kit (Genmed Scientifics Inc., Ellington, TX, USA).

The activity of complex I, II, III and IV in the mitochondrial electron transport chain was detected by chemical colorimetry (Genmed Scientifics Inc., Ellington, TX, USA). Complex I activity was measured by the oxidation of NADH by ubiquinone-1 at $340 \mathrm{~nm}$, complex II activity was measured by the oxidation of succinate by ubiquinone- 2 at $600 \mathrm{~nm}$, complex III activity was assayed by the reduction of cytochrome $\mathrm{C}$ by ubiquinol-2 at $550 \mathrm{~nm}$, and complex IV activity was assayed by the oxidation of dithionite-reduced cytochrome $\mathrm{C}$ at 550 $\mathrm{nm}$. Assays for complexes I and III were done in the presence and absence of the specific inhibitors rotenone and antimycin A, respectively, to evaluate the contribution of contaminant activities. The final concentration of total proteins was determined by the method of Lowry et al. ${ }^{32}$ The activity of the complex was expressed as the rate at which each complex in the unit protein reduced the substrate in the catalytic reaction (U/mg/min).

The measurement of mitochondrial membrane potential was performed according to the protocol of the Mitochondrial Membrane Potential Detection Kit (Genmed Scientifics Inc., Ellington, TX, USA). The mitochondrial samples were incubated with 5,5',6,6'-tetrachloro-1,1',3'tetraethylbenzimidazolcarbocyanine iodide staining working solution at room temperature for 10 minutes. Relative 
fluorescence units (RFUs) were obtained by measuring the excitation/emission wavelengths at 490/590 nm with a fluorescence microplate reader (BioTek, Synergy H4, Winooski, VT, USA). The mitochondrial membrane potential was expressed as RFU per mg of protein (RFU/mg).

\section{Oxidative Stress Markers}

As markers of oxidative stress, glutathione (GSH), and malondialdehyde (MDA) levels of the dorsal root ganglion were measured by a microplate reader (BioTek, Synergy H4, Winooski, VT, USA) using the assay kits (Nanjing Jiancheng Bioengineering Research Institute, Nanjing, China) according to the procedure recommended by the manufacturer.

\section{Apoptosis}

Neuronal apoptosis was determined using a terminal deoxynucleotidyl nick-end labeling (TUNEL) assay kit according to the manufacturer's protocol (Roche, Basel, Switzerland). The percentage of TUNEL-positive cells was calculated by dividing the number of nuclei that stained positive by the total number of nuclei in a given field of view (under $400 \times$ microscope magnification).

\section{Western Blot Analysis}

The protein of the isolated L4-5 dorsal root ganglia was extracted and separated by $8-12 \%$ sodium dodecyl sulfate polyacrylamide gel electrophoresis (SDS-PAGE) and further transferred to polyvinylidene fluoride (PVDF) membranes. After blocking with 5\% nonfat milk for $2 \mathrm{~h}$, the PVDF membranes were incubated with specific primary antibodies at $4^{\circ}$ Cover-night and washed. Membranes were incubated with secondary antibodies for $1 \mathrm{~h}$. Detection was visualized using the Odyssey Infrared Imaging System (LI-COR, Inc., Lincoln, NA, USA) and analyzed with Image J software. The basal levels of proteins were normalized to levels of $\beta$-actin protein. The primary antibodies were used in the following dilutions: SIRT1, 1:400 (Abcam, Cambridge, UK); acetyl-p53, 1:500 (Abcam, Cambridge, UK); Drp1, 1:1000 (Abcam, Cambridge, UK); BAX, 1:2000 (Protaintech, Rosemont, IL,
USA); BCL-2, 1:500 (Affinity Bioscience, Cincinnati, OH, USA); and cleaved caspase-3, 1:1000 (Cell Signaling, Danvers, MA, USA); $\beta$-actin,1:5000 (Abways, Shanghai, China).

\section{Reverse Transcription-Quantitative Polymerase Chain Reaction Analysis (qPCR)}

Total RNA was extracted from dorsal root ganglion using TRIzol (Invitrogen, Thermo Fisher Scientific, Inc., Waltham, MA, USA) according to the manufacturer's protocol. cDNA was synthesized from mRNA using a RevertAid First Strand cDNA Synthesis Kit (Thermo Fisher Scientific, Inc. Waltham, MA, USA), followed by qPCR using a SYBR ${ }^{\circledR}$ Green PCR Master Mix (Applied Biosystems; Thermo Fisher Scientific, Inc. Waltham, MA, USA) and the StepOnePlus sequence detection system (Applied Biosystems; Thermo Fisher Scientific, Inc. Waltham, MA, USA). Cycling conditions of the reactions were as follows: $10 \mathrm{~min}$ of predenaturation at $95^{\circ}$ $\mathrm{C}$ followed by 40 cycles of $15 \mathrm{~s}$ denaturation at $95^{\circ} \mathrm{C}$ and 60 s of annealing at $60{ }^{\circ} \mathrm{C}$. The sequences of the primers used are listed in Table 1 . The housekeeping gene $\beta$-actin was used for normalization.

\section{Statistical Analysis}

The statistical analysis software SPSS 24.0 was used to analyze the data, and the measurement data were described by the mean \pm standard deviation (SD). First, analysis of variance was performed for the comparison of multiple groups, and then the Tukey's test was used for multiple comparisons. Statistical tests were performed using two-sided tests, and $\mathrm{P} \leq 0.05$ was considered to indicate statistically significant differences.

\section{Results}

\section{The Fasting Blood Glucose and Weight}

As shown in Figure 1A, STZ-injected rats displayed hyperglycemia. Diabetic rats had significantly higher fasting blood glucose levels compared to rats in the control group $(p<$

Table I Primer Information for the RT-PCR

\begin{tabular}{|l|c|c|c|}
\hline Gene & Database Accession Number & Forward Primer (5'-3') & Reverse Primer (5'-3') \\
\hline SIRTI & NM_001372090.1 & AGATTTCAAGGCTGTTGGTTCC & CAGCATCATCTTCCAAGCCATT \\
$p 53$ & XM_006246594.3 & CAGCGACAGGGTCACCTAATTC & GGCAGAACAGCTTATTGAGGGA \\
DrpI & NM_053655.3 & TCCAGCTCATTACCAAGTTTGC & CAGATTCTAAGGTTCGCCCAAA \\
BAX & NM_017059.2 & GGCGATGAACTGGACAACAAC & CCCAGTTGAAGTTGCCGTCT \\
BCL-2 & NM_016993.1 & TTGTGGCCTTCTTTGAGTTCG & GCATCCCAGCCTCCGTTAT \\
$\beta$-Actin & NM_03II44 & TGCTATGTTGCCCTAGACTTCG & GTTGGCATAGAGGTCTTTACGG \\
\hline
\end{tabular}



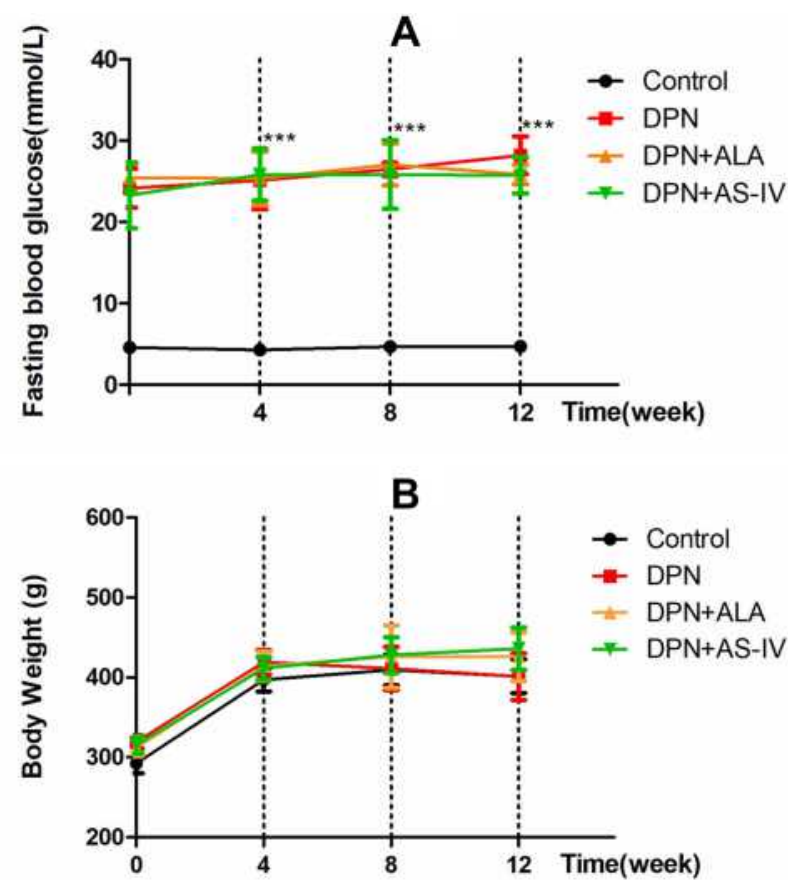

Figure I Effects of AS-IV on fasting blood glucose and body weight in diabetic rats at 12 weeks.

Notes: Fasting blood glucose levels $(\mathbf{A})$ and body weight $(\mathbf{B})$ were measured every 4 weeks. Data are represented as mean $\pm S D$. $* * * P<0.001$ vs control group. Abbreviations: SD, standard deviation; DPN, diabetic peripheral neuropathy; ASIV, astragaloside IV; ALA, $\alpha$-lipoic acid.

0.001). ALA or AS-IV treatment did not bring about any significant changes in the fasting blood glucose levels of DPN rats. As shown in Figure 1B, the weights of diabetic rats at the 4th, 8th and 12th week were not significantly different from that of the control group.

\section{Pain Thresholds}

STZ-injected rats displayed mechanical hyperalgesia. Diabetic rats were less tolerant to pressure applied to the paw as

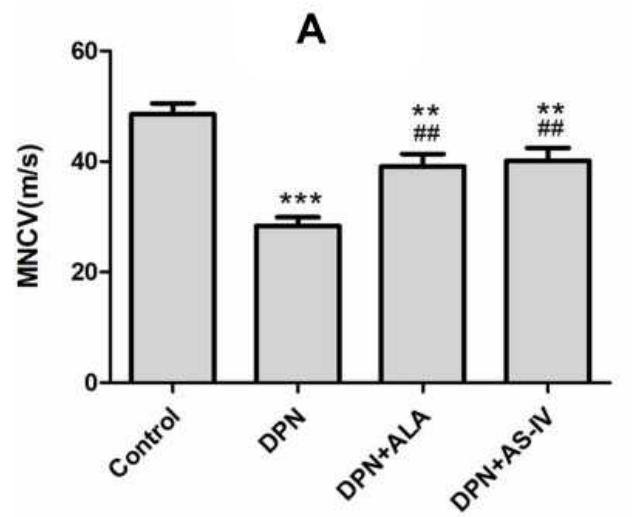

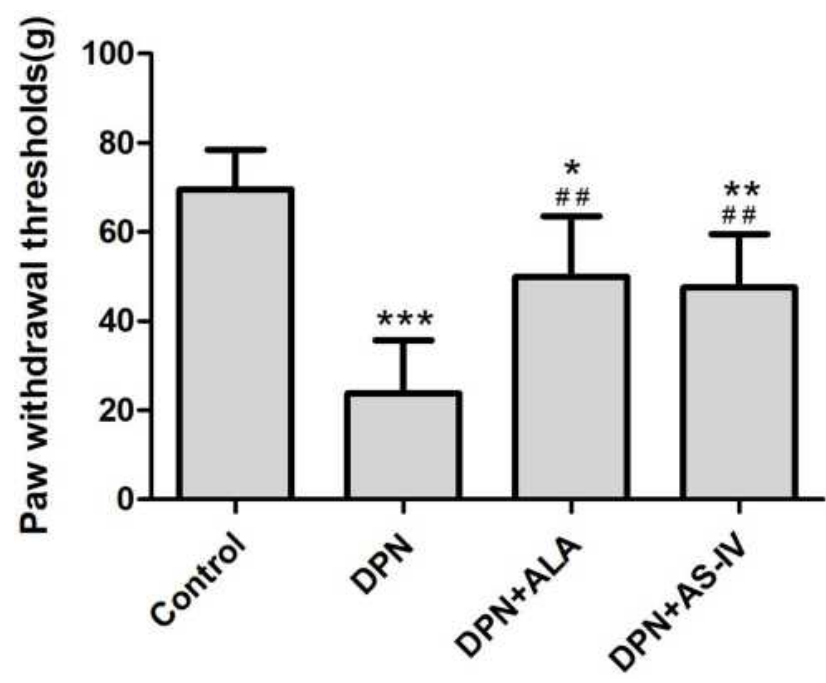

Figure 2 Effects of AS-IV on paw withdrawal thresholds.

Notes: Data are represented as mean $\pm \mathrm{SD}$. $* \mathrm{P}<0.05$, $* * \mathrm{P}<0.0 \mathrm{I}$, *** $\mathrm{P}<0.00$ I vs control group. ${ }^{\#} P<0.01$ vs DPN group.

Abbreviations: SD, standard deviation; DPN, diabetic peripheral neuropathy; ASIV, astragaloside IV; ALA, $\alpha$-lipoic acid.

compared to rats in the control group. ALA or AS-IV treatment was able to significantly reduce mechanical hyperalgesia of the hind paws of DPN rats $(\mathrm{p}<0.01)$, as shown in Figure 2 .

\section{Nerve Conduction Velocity}

The DPN group rats had significant decreases in the MNCV and SNCV compared with the control group rats. The MNCV and SNCV of the two groups of rats treated with ALA and AS-IV were higher than those in the DPN group (Figure $3 \mathrm{~A}$ and $\mathrm{B}$ ).

The decrease of NCV and the mechanical hyperalgesia validated the DPN model. ${ }^{33}$

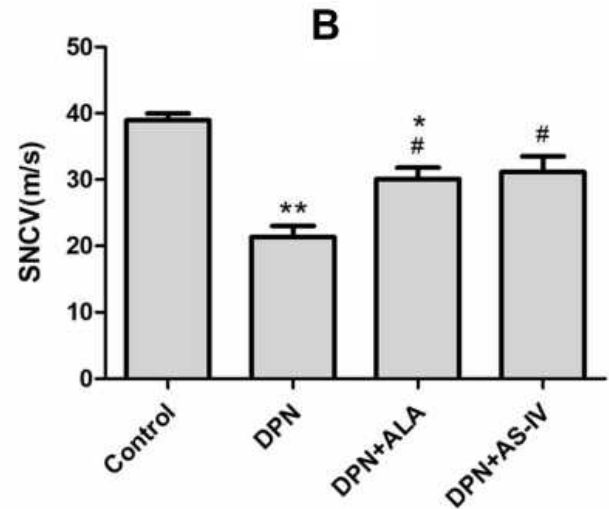

Figure 3 Effect of AS-IV on NCV.

Notes: (A) MNCV; (B) SNCV. Data are represented as mean \pm SD. $* \mathrm{P}<0.05$, ${ }^{* * P}<0.01$; ***P $<0.001$ vs control group. ${ }^{\#} \mathrm{P}<0.05$, ${ }^{\#} \mathrm{P}<0.0 \mathrm{I}$ vs $\mathrm{DPN}$ group. Abbreviations: NCV, nerve conduction velocity; MNCV, motor nerve conduction velocity; SNCV, sensory nerve conduction velocity; SD, standard deviation; DPN, diabetic peripheral neuropathy; AS-IV, astragaloside IV; ALA, $\alpha$-lipoic acid. 


\section{Mitochondrial Morphology}

In the control group, dorsal root ganglion neurons were rich in mitochondria and had normal structures. The inner and outer mitochondrial membranes and mitochondrial cristae were intact and well distributed. In the DPN group, the mitochondria of dorsal root ganglion neurons were swollen and degenerated, the outer mitochondrial membrane was damaged, and the mitochondrial cristae were broken, sparse, or had even disappeared, and most showed vacuolar changes. Mitochondria are the most destructive, with the highest MQI score (4 points). The mitochondria of DRG neurons in the DPN plus ALA and DPN plus AS-IV groups were slightly swollen, with a relatively complete structure and clear mitochondrial cristae. The MQI score of most mitochondria was 2-3. The mitochondrial damage in rats treated with ALA and AS-IV was alleviated compared with the DPN group (Figure $4 \mathrm{~A}$ and $\mathrm{B})$.

\section{Mitochondrial Function}

The measured activity of complex I, II, III, and IV in the mitochondrial electron transport chain is shown in Figure 5A-D. Complex I, II, III, and IV activities were significantly lower in the DPN than the control group $(p<0.01)$. The levels of the two groups of rats treated with ALA and AS-IV were restored and were higher than those in DPN animals $(\mathrm{p}<0.05)$. The activities of complex I, II, III of the two groups of rats treated with ALA and AS-IV were not statistically different from that of the control group, and the activity of compound IV was lower than that of the control group $(\mathrm{p}<0.05)$.

As shown in Figure 6, the mitochondrial membrane potential of the dorsal root ganglion of DPN rats was significantly decreased compared with control rats $(\mathrm{p}<$ 0.001), and that of the ALA or AS-IV treatment group also decreased $(\mathrm{p}<0.01)$. The mitochondrial membrane potential was higher in the two groups of animals treated with ALA and AS-IV than in DPN animals $(\mathrm{p}<0.05)$.

\section{Oxidative Stress Markers}

As shown in Figure 7A, the content of MDA in the dorsal root ganglia of diabetes rats was higher than that in the control group. The MDA content of the two groups of rats
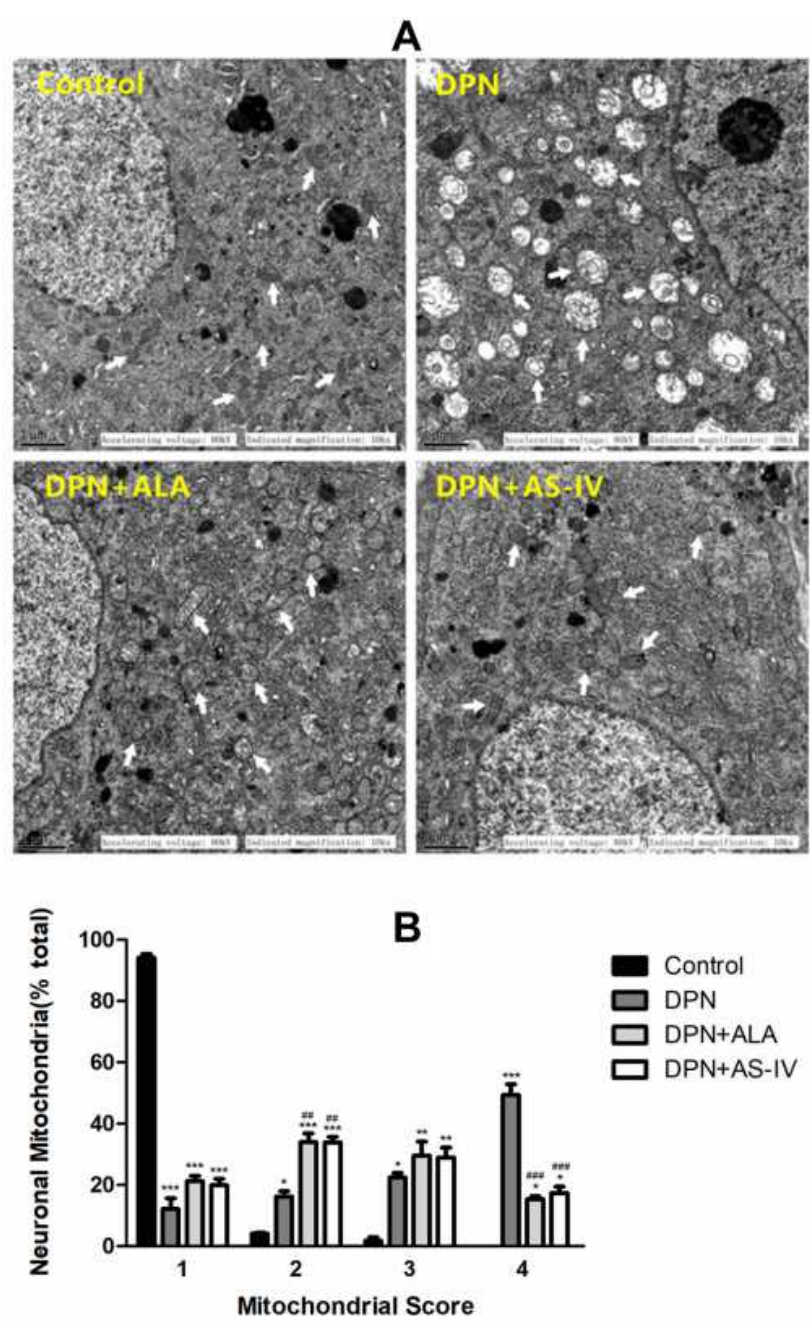

Figure 4 Effect of AS-IV on mitochondrial pathomorphology.

Notes: (A) Electron micrographs illustrated the intact mitochondria (white arrows). Scale bar, I $\mu \mathrm{m}$. (B) Mitochondria quality index score. Data are represented as mean $\pm \mathrm{SD}$. $* \mathrm{P}<0.05$, ${ }^{*} \mathrm{P} P<0.0 \mathrm{I}$, ${ }^{* * *} \mathrm{P}<0.001$ vs control group. ${ }^{\#} \mathrm{P}<$ $0.01, \ldots+0.001$ vs control group.

Abbreviations: SD, standard deviation; DPN, diabetic peripheral neuropathy; ASIV, astragaloside IV; ALA, $\alpha$-lipoic acid.

treated with ALA and AS-IV was lower than that of the DPN group $(\mathrm{p}<0.01)$.

As shown in Figure 7B, compared with the control group, the level of GSH in the dorsal root ganglia of the DPN group decreased $(\mathrm{p}<0.05)$. The GSH levels of the two groups of rats treated with ALA and AS-IV were lower than those in the DPN group $(\mathrm{p}<0.05)$.

\section{Neuronal Apoptosis}

As can be seen from Figure 8A and B, TUNEL staining micrographs showed an increased percentage of positive cells with DNA fragmentation in the DPN group $(\mathrm{p}<$ 

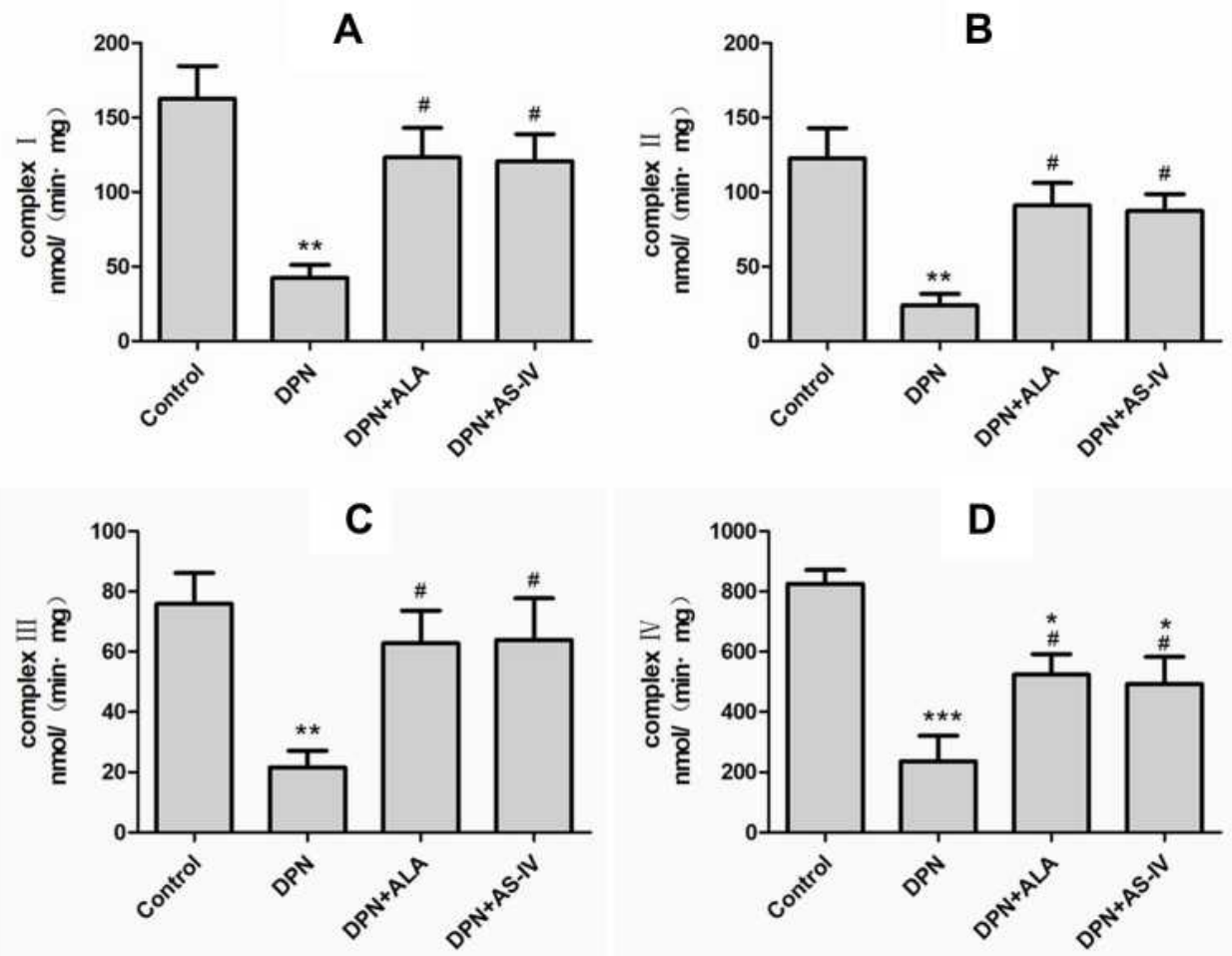

Figure 5 Effect of AS-IV on mitochondrial electron transport chain complex activity.

Notes: (A) Mitochondrial electron transport chain complex I activity. (B) Mitochondrial electron transport chain complex II activity. (C) Mitochondrial electron transport chain complex III activity. (D) Mitochondrial electron transport chain complex IV activity. Data are represented as mean $\pm \mathrm{SD}$. $* \mathrm{P}<0.05$, $* * \mathrm{P}<0.0 \mathrm{I}$, $* * * \mathrm{P}<0.00 \mathrm{I}$ vs control group. ${ }^{\#} \mathrm{P}<0.05$ vs DPN group.

Abbreviations: SD, standard deviation; DPN, diabetic peripheral neuropathy; AS-IV, astragaloside IV; ALA, $\alpha$-lipoic acid.

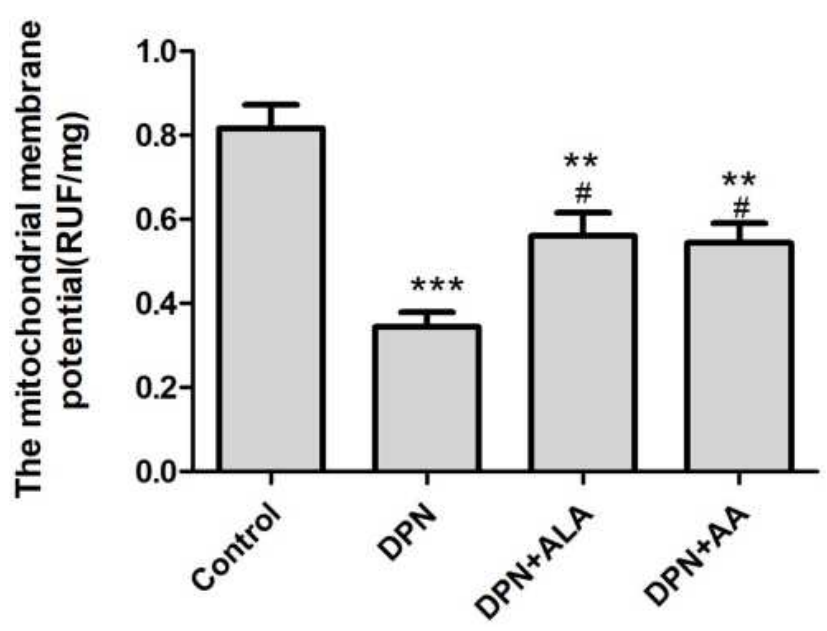

Figure 6 Effect of AS-IV on mitochondrial membrane potential.

Notes: Data are represented as mean $\pm \mathrm{SD}$. $* * \mathrm{P}<0.0 \mathrm{I}$, $* * * \mathrm{P}<0.00 \mathrm{I}$ vs control group. ${ }^{\#} \mathrm{P}<0.05$ vs DPN group.

Abbreviations: SD, standard deviation; DPN, diabetic peripheral neuropathy; ASIV, astragaloside IV; ALA, $\alpha$-lipoic acid.

0.001), with a comparatively lower percentage of positive cells with DNA fragmentation observed in the two groups of rats treated with ALA and AS-IV $(\mathrm{p}<0.05)$. As shown in Figure 8C and D, the expression of cleaved caspase- 3 in diabetic rats was significantly up-regulated compared with the control group $(\mathrm{p}<0.001)$. Compared with the DPN group, the expression of cleaved caspase-3 was downregulated in the two groups of rats treated with ALA and AS-IV $(\mathrm{p}<0.001)$.

\section{SIRTI/p53 Signaling Pathway}

The expression of related proteins in the SIRT1/p53 signaling pathway was evaluated by IHC. The positive expression of SIRT1 was mainly observed in the nucleus and partly in the cytoplasm. Acetyl-p53, Drp1, BAX, and BCL-2 were observed in the cytoplasm (Figure 9).

As shown in Figure 10A and B, Western blot analysis showed that the expression of SIRT1 in the two groups of rats treated with ALA and AS-IV was significantly up-regulated compared with the DPN group $(p<0.05)$. As shown in Figure 10A and C, compared with the control group, the expression of acetyl-p53 in diabetic rats was significantly up-regulated $(p<0.001)$. The expression of acetyl-p53 in the two groups treated 

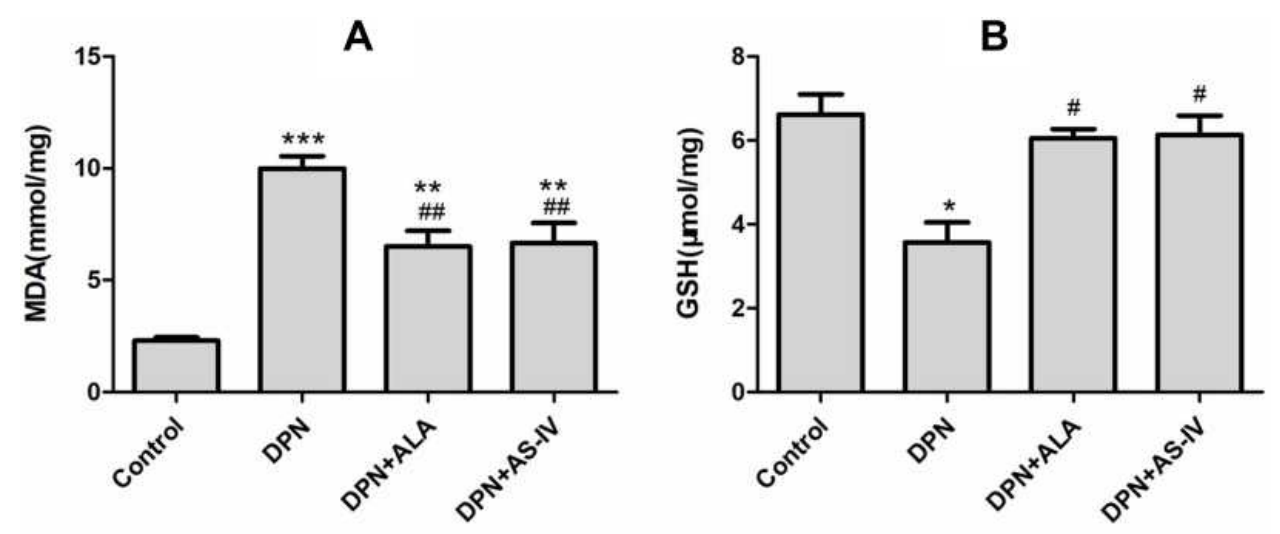

Figure 7 Effect of AS-IV on oxidative stress.

Notes: (A) MDA, (B) GSH. Data are represented as mean \pm SD. $* \mathrm{P}<0.05$, **P $<0.01$; ${ }^{* * * P}<0.00$ I vs control group. ${ }^{\#} \mathrm{P}<0.05$, ${ }^{\#} \mathrm{P}<0.0 \mathrm{I}$ vs $\mathrm{DPN}$ group. Abbreviations: MDA, malonaldehyde; GSH, glutathione; SD, standard deviation; DPN, diabetic peripheral neuropathy; AS-IV, astragaloside IV; ALA, $\alpha$-lipoic acid.

with ALA and AS-IV was significantly lower than that in the DPN group. As shown in Figure 10A and D, the expression of Drp1 in DPN group and ALA group was higher than that in the control group. Compared with the DPN group, the expression of Drp1 was downregulated in the two groups treated with ALA and ASIV. Analysis of the ratio of BAX to BCL-2 showed that the ratio in the DPN group was higher than that in the control group $(p<0.001)$. Compared with the DPN group, the ratio of $\mathrm{BAX}$ to $\mathrm{BCL}-2$ in the two groups treated with ALA and AS-IV decreased (Figure 10A and $\mathrm{E})$.

To explore the underlying mechanism of AS-IV inhibiting cell apoptosis, the expression of genes involved in the SIRT1/p53 pathway was assessed. The expression of SIRT1 in the two groups of rats treated with ALA and ASIV was significantly up-regulated compared with the DPN group ( $\mathrm{p}<0.01$ ) (Figure 11A). Compared with the control group, the expression of p53 in the DPN group was upregulated $(\mathrm{p}<0.001)$. The $\mathrm{p} 53$ expression of the two groups of rats treated with ALA and AS-IV was lower than that of the DPN group ( $<<0.001)$. (Figure 11B) Compared with the control group, the expression of Drp1 in the DPN group was significantly up-regulated ( $p$ $<0.001)$. The expression of Drp1 in the two groups of rats treated with ALA and AS-IV was significantly lower than that in the DPN group ( $<<0.001)$ (Figure 11C). The expressions of BAX and BCL-2 in diabetic rats were higher than those in the control group. Compared with the DPN group, the expression of BAX in the two groups of rats treated with ALA and AS-IV was significantly down-regulated, while the expression of BCL-2 was upregulated $(\mathrm{p}<0.001)$ (Figure $11 \mathrm{D}$ and $\mathrm{E})$.

\section{Discussion}

The main purpose of the experiment was to explore the therapeutic effect of AS-IV on DPN rats and its molecular mechanism. The current study evaluated the effect of AS-IV on the development of DPN rats induced by STZ. We found that AS-IV has a neuroprotective effect similar to ALA. Both of them can significantly alleviates hyperalgesia, increase nerve conduction velocity, and play a therapeutic role by regulating the SIRT1/p53 pathway to reduce mitochondria-dependent apoptosis.

Neuropathic pain is the most common impediment associated with DPN. Thus, measurement of allodynia and hyperalgesia in response to mechanical stimuli is considered an important, reliable, and sensitive index for neuropathic pain during DPN. ${ }^{34}$ An array of researchers used various methods to evaluate allodynia and hyperalgesia during the experimental DPN, such as von Frey filaments test (Paw withdrawal response to light touch of plantar surface), Hargreaves method (Paw withdrawal response latency to escalating heat applied to the plantar surface), Paw intradermal formalin-evoked responses, Randall-Selitto test, etc. ${ }^{34-36}$ In the present investigation, the results of the von Frey 
A
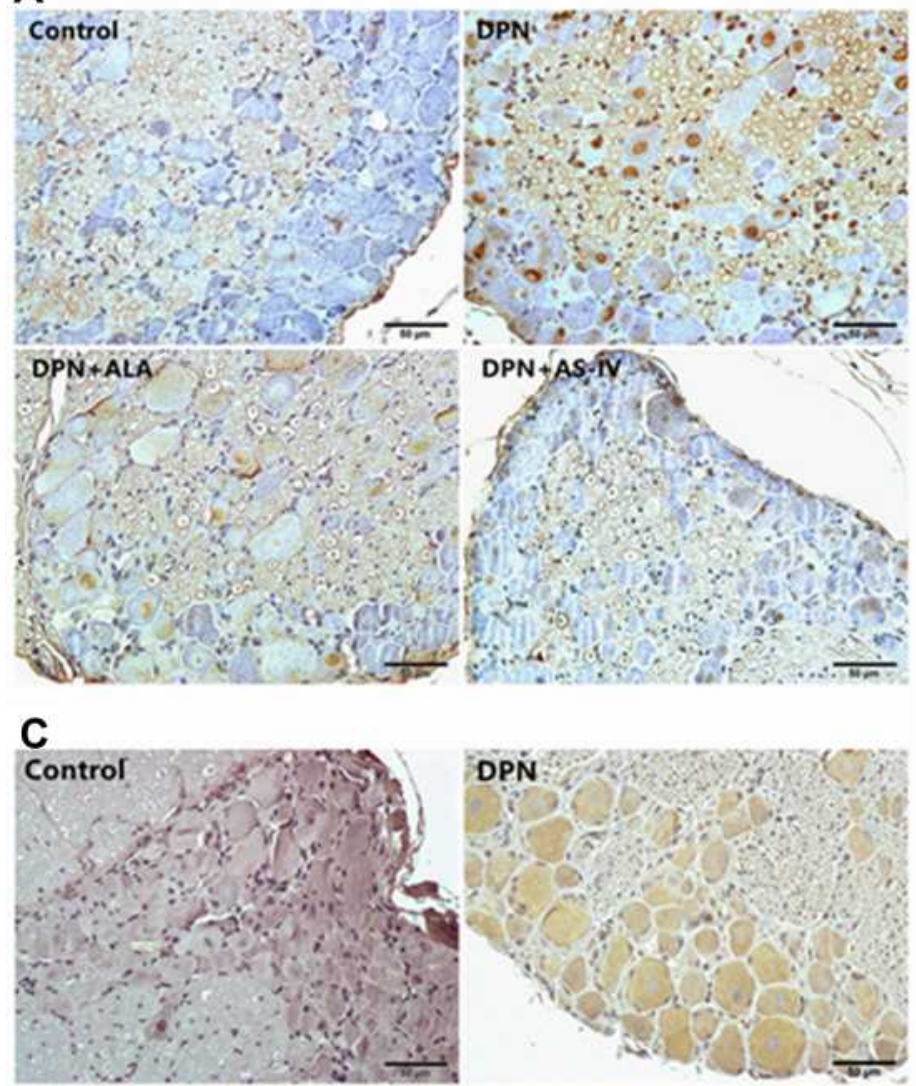

DPN+ÁLA

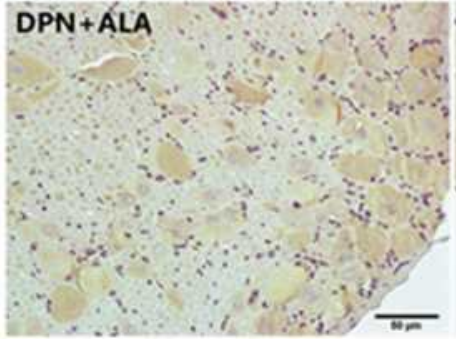

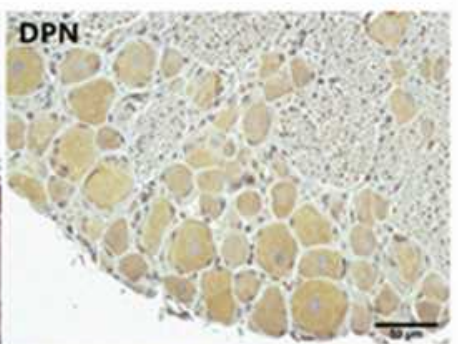

DPN+AS-IV

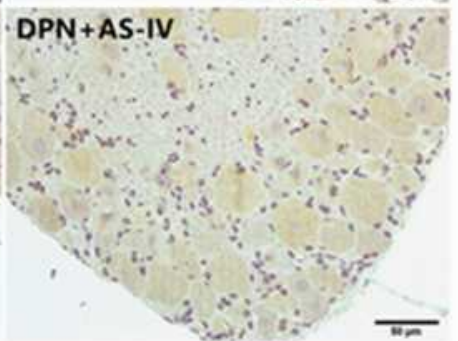

B
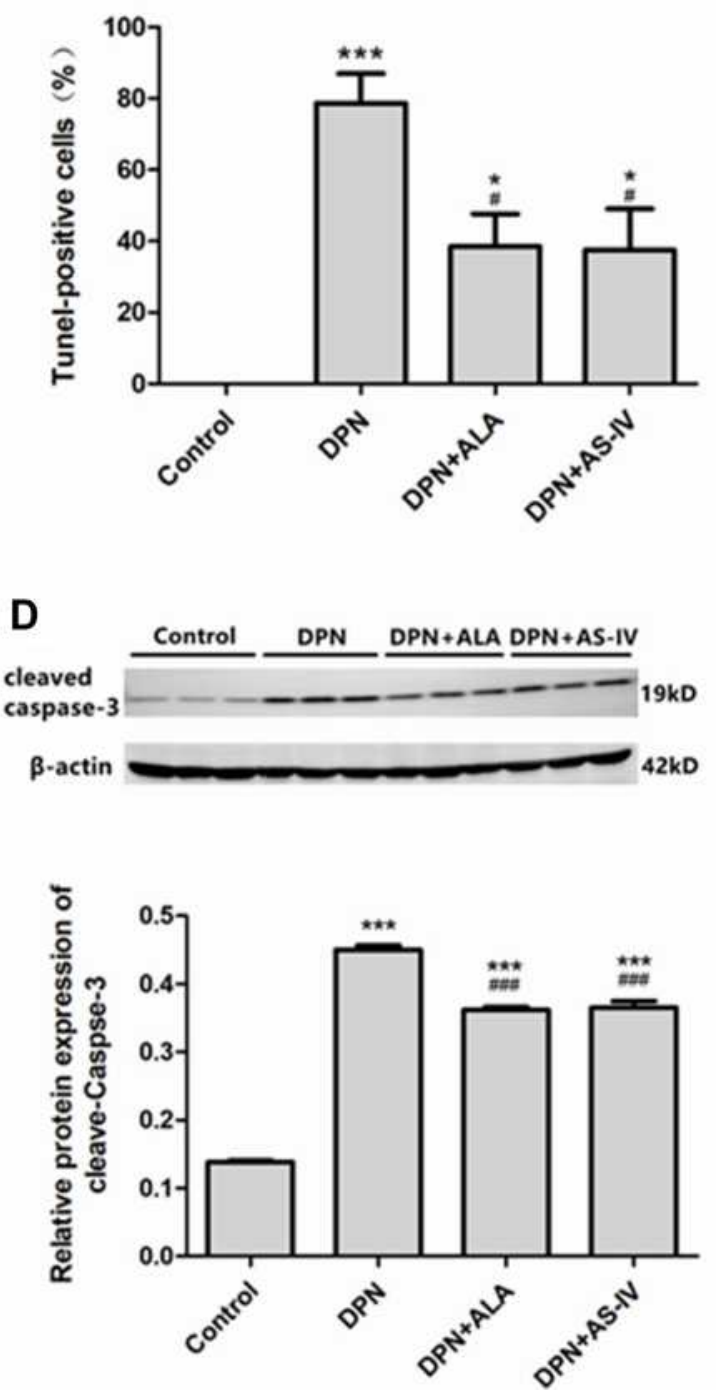

Figure 8 Effect of AS-IV on apoptosis.

Notes: (A and B) The results of TUNEL staining. Scale bar, $50 \mu \mathrm{m}$. (C) Immunohistochemical staining of cleaved caspase-3. Scale bar, $50 \mu \mathrm{m}$. (D) The cleaved caspase-3

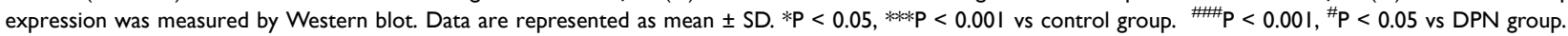
Abbreviations: TUNEL, terminal deoxynucleotidyl nick-end labeling; SD, standard deviation; DPN, diabetic peripheral neuropathy; AS-IV, astragaloside IV; ALA, $\alpha$-lipoic acid.

filaments test showed that the mechanical pain threshold of the DPN group was reduced, confirming the neuropathic pain of DPN, and the treatment of AS-IV and ALA can increase the mechanical pain threshold of DPN rats and reduce allodynia and hyperalgesia.

Electrophysiological assessment of nerve function in diabetic patients provides a quantitative measure of nerve integrity and a means to monitor disease onset and progression. $^{37}$ In rodent models of diabetic neuropathy, NCV measurements are commonly used to define the extent of nerve dysfunction as well as providing an endpoint in studies of putative treatments. $^{33}$ Our research results showed that the NCV of the DPN group decreased, which proved that STZ and high-carbohydrate/high-fat diet-induced diabetic rats had obvious neurological dysfunction. Interestingly, AS-IV and ALA can improve MNCV and SNCV in DPN rats, and their effects are similar. 


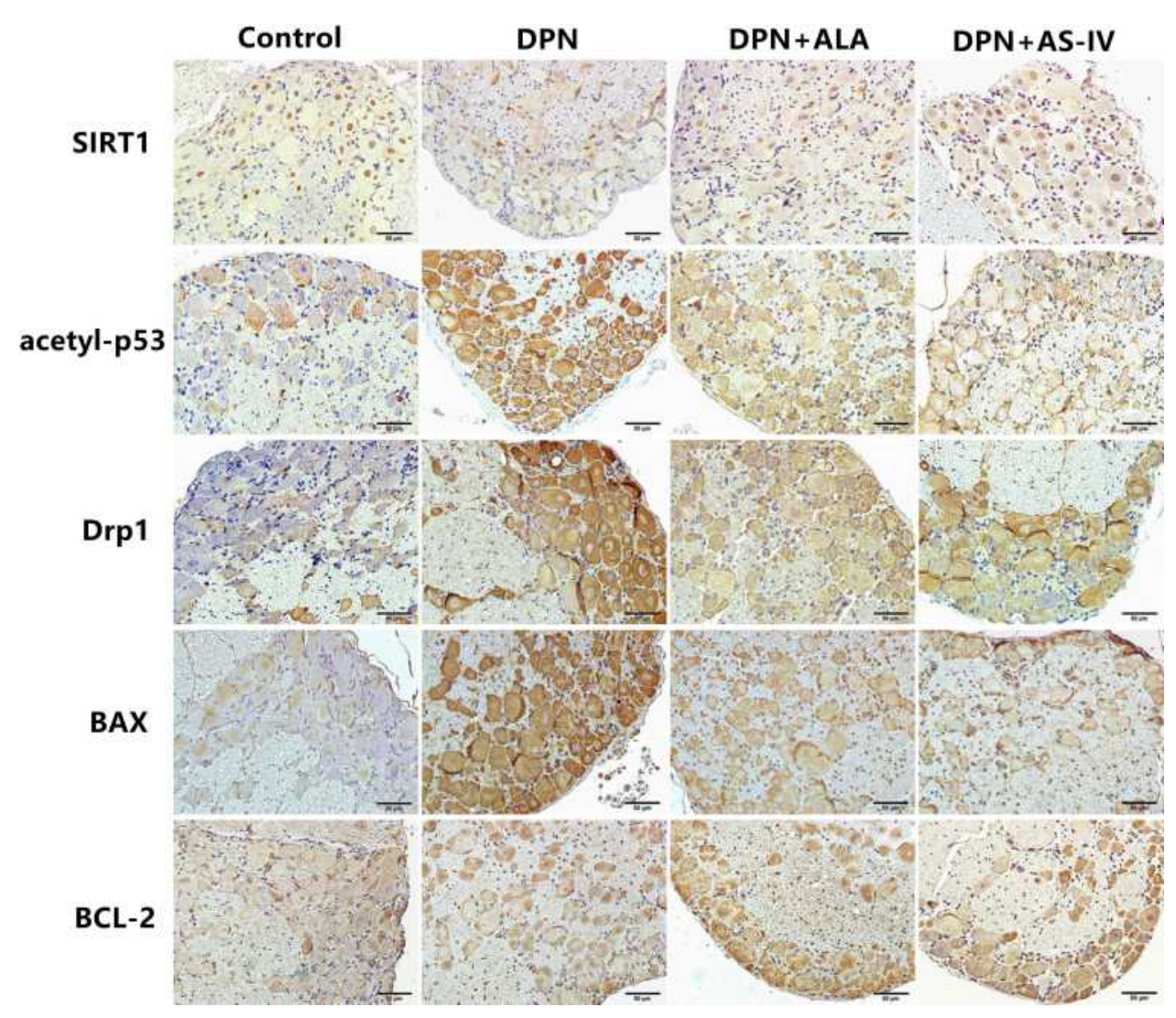

Figure 9 Immunohistochemical staining of SIRTI, acetyl-p53, DrpI, BAX, BCL-2. Scale bar: $50 \mu \mathrm{m}$. Abbreviations: DPN, diabetic peripheral neuropathy; AS-IV, astragaloside IV; ALA, $\alpha$-lipoic acid.

Some evidence suggests that mitochondria play an important role in sensory neurons in the pathophysiology of abnormal pain. ${ }^{38-40}$ In nerve cells, the position of mitochondria is particularly important. Mitochondria provide energy for various activities of neurons and are almost the exclusive providers of energy. Mitochondria are considered to be a key regulator of neuron-related complications in diabetes. ${ }^{41} \mathrm{~A}$ decrease in mitochondrial membrane potential is the key event initiating mitochondrial-dependent apoptosis. We observed that the mitochondria of DRG neurons in DPN rats were swollen and vacuolated, and the mitochondrial cristae disappeared. At the same time, the activity of complex I-IV in the mitochondrial electron transport chain and the mitochondrial membrane potential decreased, the expression of cleaved caspase-3 up-regulated, and the number of apoptotic cells increased. These are consistent with the results of other researchers. ${ }^{6,7,42}$ AS-IV and ALA treatment can significantly reduce the structural damage of mitochondria, enhance the activity of the mitochondrial electron transport chain complex, protect and maintain the mitochondrial membrane potential, and improve mitochondrial function, thereby reducing cell apoptosis.

p53 is induced in damaged neurons and has a neurotoxic function in animal models of neurodegenerative disease. ${ }^{43}$ p53 can cause the excessive fission of mitochondria mediated by Drp1. Excessive fission severely damages the mitochondrial genome, resulting in damage of the mtDNA that encodes the 13 polypeptides involved in oxidative phosphorylation and mitochondrial structure, ${ }^{44}$ opening of the mitochondrial permeability transition pore (mPTP), and increased reactive oxygen species (ROS) production. ${ }^{45}$ p53 can also reduce the mitochondrial membrane potential through the pro-apoptotic protein BAX, increase mitochondrial membrane permeability, release mitochondrial-related pro-apoptotic factors, and initiate the caspase cascade reaction to induce 

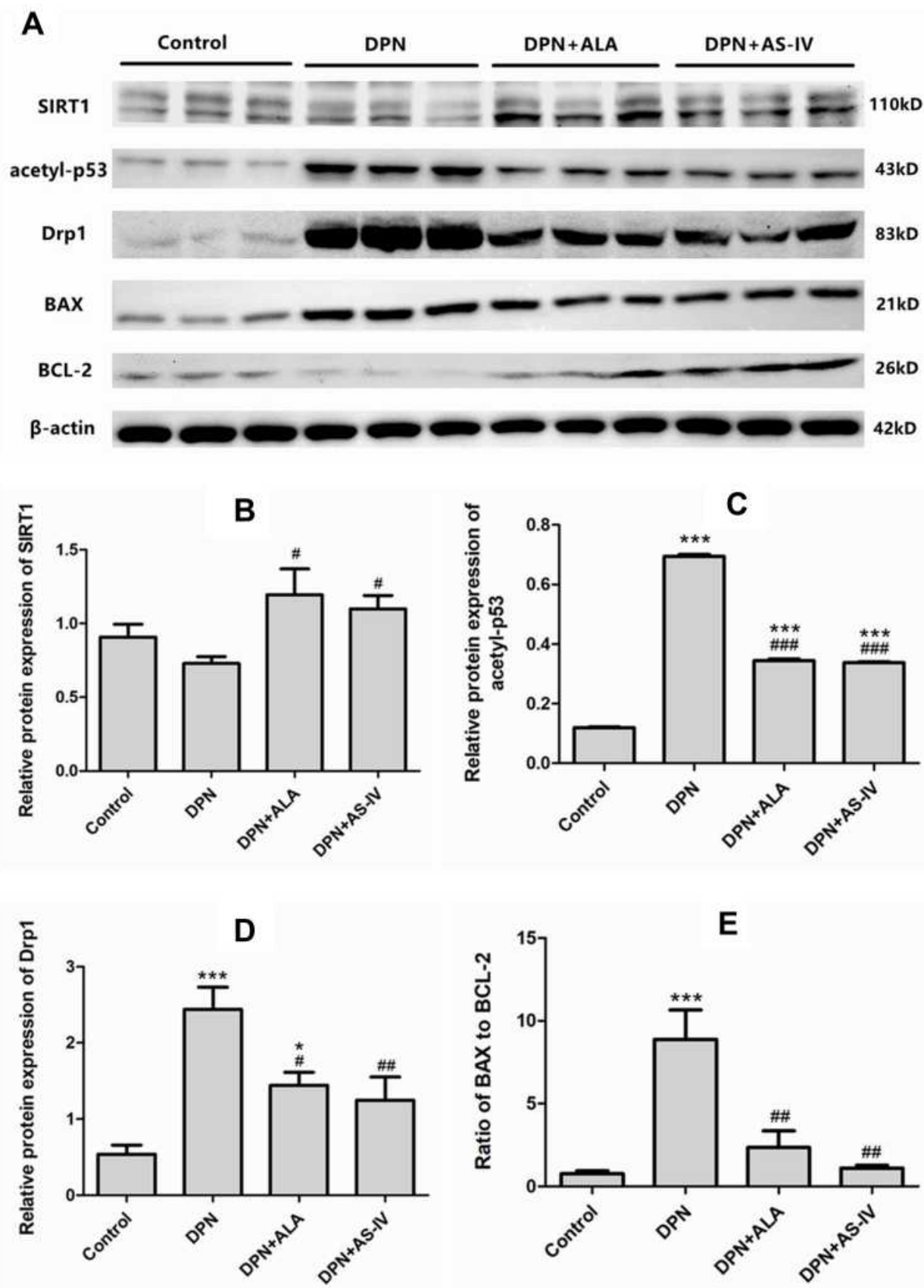

Figure 10 Western blot analysis of SIRTI, acetyl-p53, DrpI, BAX, BCL-2.

Notes: (A) Films of SIRTI, acetyl-p53, DrpI, BAX, BCL-2, and $\beta$-actin bands. (B-D) Levels of SIRTI (B), acetyl-p53 (C), DrpI (D) expressed as the optical density value normalized to $\beta$-actin. (E) Ratio of optical density value of $B A X$ to $B C L-2$. Data are represented as mean $\pm S D$. $* P<0.05, * * * P<0.001$ vs control group. ${ }^{\# P}<0.05$, ${ }^{\# \#} P<$ $0.01,{ }^{\prime \prime} P 0.001$ vs DPN group.

Abbreviations: SD, standard deviation; DPN, diabetic peripheral neuropathy; AS IV, astragaloside IV; ALA, $\alpha$-lipoic acid.

apoptosis. $^{46,47}$ In this study, acetyl-p53 was significantly expressed in DPN rats, downstream Drp1 and BAX expression was upregulated, and anti-apoptotic protein BCL-2 expression was downregulated. This suggests that apoptosis induced by p53-mediated mitochondrial destruction is an important molecular mechanism leading to DPN.

SIRT1 acts as a negative regulator of p53 to inhibit cell apoptosis. ${ }^{48}$ The data of Shi et al showed that high concentrations of glucose can induce hippocampal 

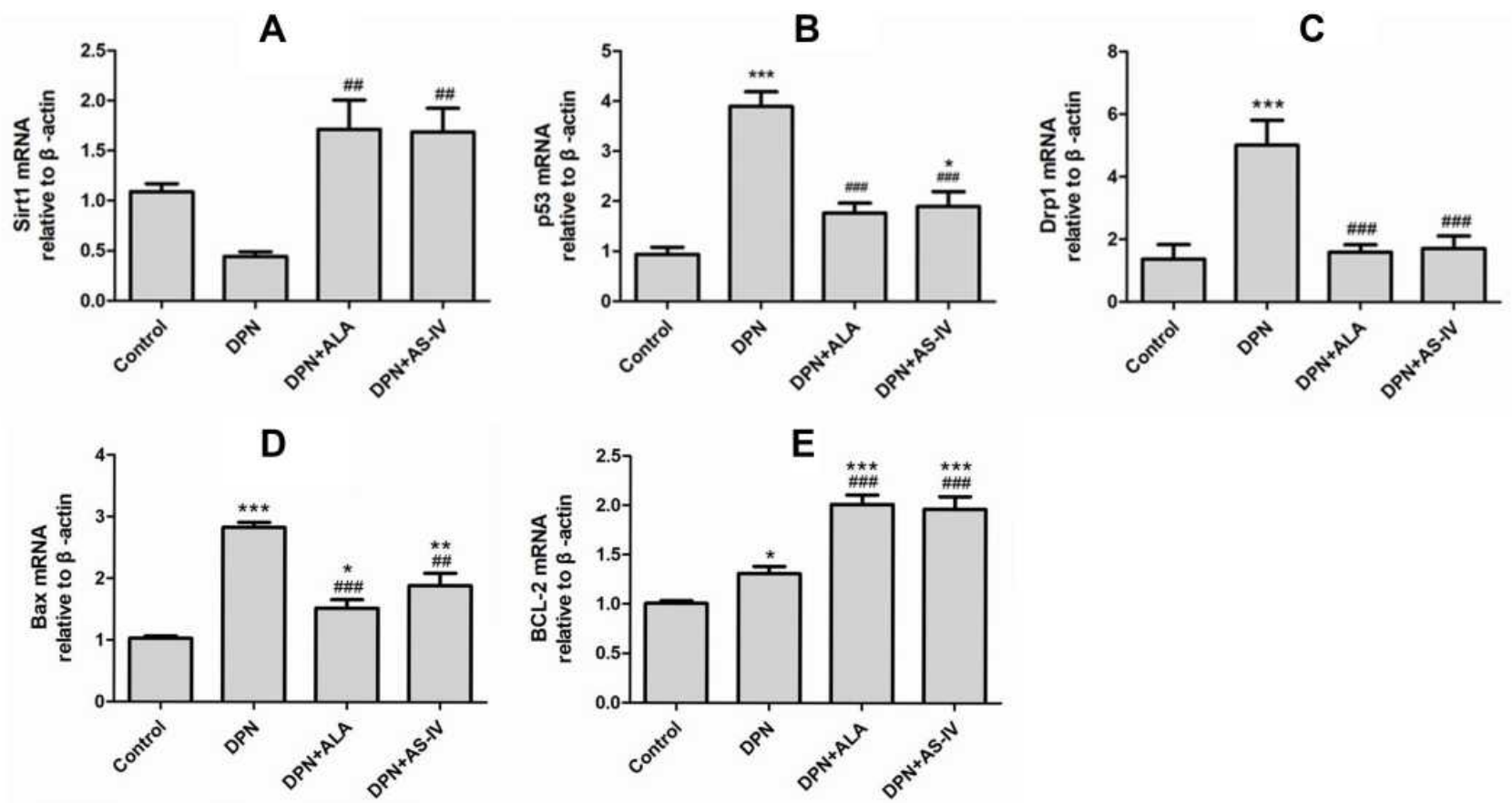

Figure I I mRNA expression of SIRTI, acetyl-p53, DrpI, BAX, BCL-2.

Notes: (A) SIRTI mRNA expression, (B) p53 mRNA expression, (C) Drpl mRNA expression, (D) BAX mRNA expression, (E) BCL-2 mRNA expression. Data are

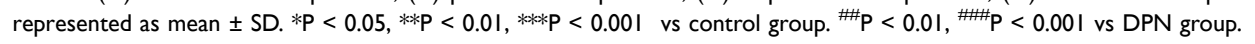

Abbreviations: SD, standard deviation; DPN, diabetic peripheral neuropathy; AS IV, astragaloside IV; ALA, $\alpha$-lipoic acid.

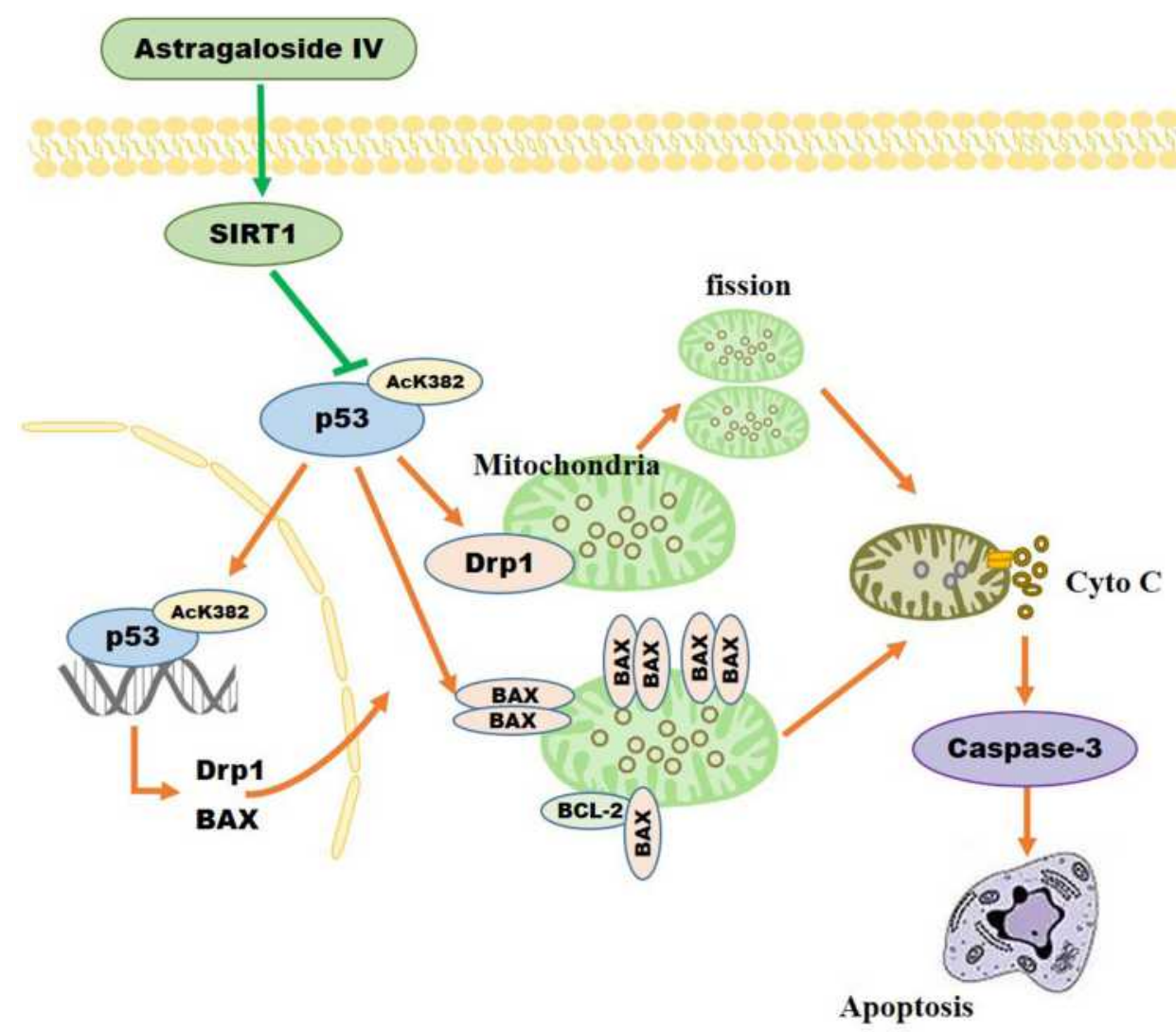

Figure I 2 As-IV inhibits mitochondrial-dependent apoptosis through modulation of the SIRTI/p53 signaling pathway. 
neuronal apoptosis by downregulating SIRT1 and increasing p53 acetylation. ${ }^{49}$ Studies have shown that ALA can activate SIRT1 and protect cardiomyocytes. ${ }^{50}$ In our study, SIRT1 expression was up-regulated in DPN rats treated with AS-IV and ALA, indicating that SIRT1 is a neuroprotective target for AS-IV and ALA. The down-regulation of acetyl-p53 and Drp1 and the ratio of $\mathrm{BAX}$ to $\mathrm{BCL}-2$ further confirmed that $\mathrm{AS}$ IV and ALA can increase mitochondrial bioenergy and mitochondrial membrane potential through the SITR1/ p53 pathway, and partially reverse the neuronal apoptosis induced by hyperglycemia.

ALA has been examined quite extensively as a treatment for diabetic neuropathy. Moreover, ALA was effective in the treatment of distal sensory motor neuropathy, as well as in the modulation of peripheral neuropathy and pain reduction in diabetic patients. ${ }^{51}$ Experimental evidence suggested that ALA could restore the glutathione levels, prevent lipid peroxidation, increase the activity of antioxidant enzymes (such as catalase and superoxide dismutase in peripheral nerves), increase the blood flow, along with $\mathrm{NCV}^{52-54} \mathrm{AS}-\mathrm{IV}$ is a natural bioactive compound isolated from Astragalus membranaceus Bunge, regarded as the critical marker to evaluate the quality of Astragalus membranaceus in the Chinese Pharmacopeia. Studies have found that AS-IV can prevent mitochondrial dysfunction of central neuron by increasing superoxide dismutase (SOD) and GSH, reducing MDA, and inhibiting oxidative stress. ${ }^{55-57}$ In our experiments, both ALA and AS-IV reduced the MDA content in the dorsal root ganglia of DPN rats and increased the GSH level, and there was no significant difference in their antioxidant effects. At the same time, they have similar functions of protecting mitochondria and inhibiting neuronal apoptosis.

\section{Conclusion}

Our results showed that AS-IV can protect mitochondrial function and structure through the SIRT1/p53 pathway, reduce cell apoptosis, and protect peripheral nerves in diabetic rats (Figure 12). It has a similar therapeutic effect as ALA and is therefore a promising drug for the potential treatment of DPN.

\section{Institutional Review Board Statement}

The study was approved by the Ethics Committee of Hebei University of Chinese Medicine (protocol code DWLL2018042, Dec 28, 2018).

\section{Data Sharing Statement}

The datasets used and/or analyzed during the current study are available from the corresponding author on reasonable request.

\section{Acknowledgments}

We are grateful to Ming He and Weisong Duan for experimental technical advices.

\section{Funding}

This research was funded by the National Natural Science Foundation of China [grant number 81803922].

\section{Disclosure}

The authors declare no conflicts of interest. The funders had no role in the design of the study; in the collection, analyses, or interpretation of data; in the writing of the manuscript, or in the decision to publish the results.

\section{References}

1. Stratton IM, Adler AI, Neil HAW, et al. Association of glycaemia with macrovascular and microvascular complications of type 2 diabetes (UKPDS35): prospective observational study. BMJ. 2000;321 (7258):405-412. doi:10.1136/bmj.321.7258.405

2. Feldman EL, Bennett DL, Nave KA, et al. New horizons in diabetic neuropathy: mechanisms, bioenergetics, and pain. Neuron. 2017;93 (6):1296-1313. doi:10.1016/j.neuron.2017.02.005

3. Diabetes Control and Complications Trial Research Group. The effect of intensive treatment of diabetes on the development and progression of long-term complications in insulin-dependent diabetes mellitus. $N$ Engl $J \quad$ Med. 1993;329(14):977-986. doi:10.1056/ NEJM199309303291401

4. Diabetes Control and Complications Trial, Epidemiology of Diabetes Interventions and Complications Research Group. Sustained effect of intensive treatment of type 1 diabetes mellitus on development and progression of diabetic nephropathy: the epidemiology of diabetes interventions and complications (EDIC) study. JAMA. 2003;290 (16):2159-2167. doi:10.1001/jama.290.16.2159

5. Ang L, Jaiswal M, Martin C, Pop-Busui R. Glucose control and diabetic neuropathy: lessons from recent large clinical trials. Curr Diab Rep. 2014;14(9):528. doi:10.1007/s11892-014-0528-7

6. Chandrasekaran K, Anjaneyulu M, Inoue T, et al. Mitochondrial transcription factor A regulation of mitochondrial degeneration in experimental diabetic neuropathy. Am J Physiol Endocrinol Metab. 2015;309 (2):E132-E141. doi:10.1152/ajpendo.00620.2014

7. Chandrasekaran K, Muragundla A, Demarest TG, et al. mGluR2/3 activation of the SIRT1 axis preserves mitochondrial function in diabetic neuropathy. Ann Clin Transl Neurol. 2017;4(12):844-858. doi:10.1002/acn3.484

8. Fernyhough P. Mitochondrial dysfunction in diabetic neuropathy: a series of unfortunate metabolic events. Curr Diab Rep. 2015;15 (11):89. doi:10.1007/s11892-015-0671-9

9. Sifuentes-Franco S, Pacheco-Moisés FP, Rodríguez-Carrizalez AD, et al. The role of oxidative stress, mitochondrial function, and autophagy in diabetic polyneuropathy. J Diabetes Res. 2017;2017:1673081. doi: $10.1155 / 2017 / 1673081$ 
10. Santiago JA, Potashkin JA. Shared dysregulated pathways lead to Parkinson's disease and diabetes. Trends Mol Med. 2013;19 (3):176-186. doi:10.1016/j.molmed.2013.01.002

11. Quijano C, Trujillo M, Castro L, et al. Interplay between oxidant species and energy metabolism. Redox Biol. 2016;8:28-42. doi:10.1016/j.redox.2015.11.010

12. Dashzeveg N, Yoshida K. Cell death decision by p53 via control of the mitochondrial membrane. Cancer Lett. 2015;367(2):108-112. doi:10.1016/j.canlet.2015.07.019

13. Ni HM, Williams JA, Ding WX. Mitochondrial dynamics and mitochondrial quality control. Redox Biol. 2015;4:6-13. doi:10.1016/j. redox.2014.11.006

14. Langley E, Pearson M, Faretta M, et al. Human SIR2 deacetylates p53 and antagonizes PML/p53-induced cellular senescence. EMBO J. 2002;21(10):2383-2396. doi:10.1093/emboj/21.10.2383

15. Vaziri H, Dessain SK, Ng Eaton E, et al. hSIR2(SIRT1) functions as an NAD-dependent p53 deacetylase. Cell. 2001;107(2):149-159. doi:10.1016/s0092-8674(01)00527-x

16. Tang Y, Zhao W, Chen Y, et al. Acetylation is indispensable for p53 activation. Cell. 2008;133(4):612-626. doi:10.1016/j. cell.2008.03.025

17. Feng L, Lin T, Uranishi H, et al. Functional analysis of the roles of posttranslational modifications at the p53 $\mathrm{C}$ terminus in regulating p53 stability and activity. Mol Cell Biol. 2005;25(13):5389-5395. doi:10.1128/MCB.25.13.5389-5395.2005

18. Ota H, Akishita M, Eto $M$, et al. Sirt1 modulates premature senescence-like phenotype in human endothelial cells. $J$ Mol Cell Cardiol. 2007;43(5):571-579. doi:10.1016/j.yjmcc.2007.08.008

19. Yi J, Luo J. SIRT1 and p53, effect on cancer, senescence and beyond. Biochim Biophys Acta. 2010;1804(8):1684-1689. doi:10.1016/j. bbapap.2010.05.002

20. Kume S, Haneda M, Kanasaki K, et al. Silent information regulator 2 (SIRT1) attenuates oxidative stress-induced mesangial cell apoptosis via p53 deacetylation. Free Radic Biol Med. 2006;40(12):2175-2182. doi:10.1016/j.freeradbiomed.2006.02.014

21. Xia ML, Xie XH, Ding JH, et al. Astragaloside IV inhibits astrocyte senescence: implication in Parkinson's disease. J Neuroinflammation. 2020;17(1):105. doi:10.1186/s12974-020-01791-8

22. Sun Q, Jia N, Wang W, et al. Protective effects of astragaloside IV against amyloid Beta1-42 neurotoxicity by inhibiting the mitochondrial permeability transition pore opening. PLoS One. 2014;9(6): e98866. doi:10.1371/journal.pone.0098866

23. Chan WS, Durairajan SS, Lu JH, et al. Neuroprotective effects of Astragaloside IV in 6-hydroxydopamine-treated primary nigral cell culture. Neurochem Int. 2009;55(6):414 422. doi:10.1016/j. neuint.2009.04.012

24. Li X, Wang X, Han C, et al. Astragaloside IV suppresses collagen production of activated hepatic stellate cells via oxidative stress-mediated p38 MAPK pathway. Free Radic Biol Med. 2013;60:168-176. doi:10.1016/j.freeradbiomed.2013.02.027

25. Li XZ, Ding YZ, Wu HF, et al. Astragaloside IV prevents cardiac remodeling in the apolipoprotein E-deficient mice by regulating cardiac homeostasis and oxidative stress. Cell Physiol Biochem. 2017;44 (6):2422-2438. doi:10.1159/000486166

26. Ziegler D, Low PA, Litchy WJ, et al. Efficacy and safety of antioxidant treatment with $\alpha$-lipoic acid over 4 years in diabetic polyneuropathy: the NATHAN 1 trial. Diabetes Care. 2011;34(9):2054-2060. doi: $10.2337 / \mathrm{dc} 11-0503$

27. Zhang BY, Zhang YL, Sun Q, et al. Alpha-lipoic acid downregulates TRPV1 receptor via NF- $\kappa B$ and attenuates neuropathic pain in rats with diabetes. CNS Neurosci Ther. 2020;26(7):762-772. doi:10.1111/ cns. 13303

28. Chen WL, Kang CH, Wang SG, Lee HM. $\alpha$-Lipoic acid regulates lipid metabolism through induction of sirtuin 1 (SIRT1) and activation of AMP-activated protein kinase. Diabetologia. 2012;55 (6):1824-1835. doi:10.1007/s00125-012-2530-4
29. Valdecantos MP, Pérez-Matute P, González-Muniesa P, et al. Lipoic acid improves mitochondrial function in nonalcoholic steatosis through the stimulation of sirtuin 1 and sirtuin 3. Obesity. 2012;20 (10):1974-1983. doi:10.1038/oby.2012.32

30. Ueda $\mathrm{H}$, Neyama H. LPA1 receptor involvement in fibromyalgia-like pain induced by intermittent psychological stress, empathy. Neurobiol Pain. 2017;1:16-25. doi:10.1016/j.ynpai.2017.04.002

31. Schwasinger-Schmidt T, Robbins DC, Williams SJ, et al. Long-term liraglutide treatment is associated with increased insulin content and secretion in $\beta$-cells, and a loss of $\alpha$-cells in ZDF rats. Pharmacol Res. 2013;76:58-66. doi:10.1016/j.phrs.2013.07.005

32. Lowry OH, Rosebrough NJ, Farr AL, et al. Protein measurement with the Folin phenol reagent. J Biol Chem. 1951;193(1):265-275. doi:10.1016/S0021-9258(19)52451-6

33. Biessels GJ, Bril V, Calcutt NA, et al. Phenotyping animal models of diabetic neuropathy: a consensus statement of the diabetic neuropathy study group of the EASD (Neurodiab). J Peripher Nerv Syst. 2014;19 (2):77-87. doi:10.1111/jns5.12072

34. Zhou G, Yan M, Guo G, Tong N. Ameliorative effect of berberine on neonatally induced Type 2 diabetic neuropathy via modulation of BDNF, IGF-1, PPAR- $\gamma$, and AMPK Expressions. Dose Response. 2019;17(3):1559325819862449. doi:10.1177/1559325819862449

35. Kandhare AD, Raygude KS, Ghosh P, Ghule AE, Bodhankar SL. Neuroprotective effect of naringin by modulation of endogenous biomarkers in streptozotocin induced painful diabetic neuropathy. Fitoterapia. 2012;83(4):650-659. doi:10.1016/j.fitote.2012.01.010

36. Visnagri A, Kandhare AD, Chakravarty S, Ghosh P, Bodhankar SL. Hesperidin, a flavanoglycone attenuates experimental diabetic neuropathy via modulation of cellular and biochemical marker to improve nerve functions. Pharm Biol. 2014;52(7):814-828. doi:10.3109/ 13880209.2013.870584

37. Tesfaye S, Boulton AJ, Dyck PJ, et al. Diabetic neuropathies: update on definitions, diagnostic criteria, estimation of severity, and treatments. Toronto Diabetic Neuropathy Expert Group. Diabetes Care. 2010;33(10):2285-2293. doi:10.2337/dc10-1303

38. Zhan L, Li R, Sun Y, et al. Effect of mito-TEMPO, a mitochondria targeted antioxidant, in rats with neuropathic pain. Neuroreport. 2018;29(15):1275-1281. doi:10.1097/WNR.0000000000001105

39. Wu P, Chen Y. Evodiamine ameliorates paclitaxel-induced neuropathic pain by inhibiting inflammation and maintaining mitochondrial anti-oxidant functions. Hum Cell. 2019;32(3):251-259. doi:10.1007/ s13577-019-00238-4

40. Sun L, Xu Q, Zhang W, et al. The involvement of spinal annexin A10/NF- $\mathrm{kB} / \mathrm{MMP}-9$ pathway in the development of neuropathic pain in rats. BMC Neurosci. 2019;20(1):28. doi:10.1186/s12868-0190513-9

41. Fernyhough P, Huang TJ, Verkhratsky A. Mechanism of mitochondrial dysfunction in diabetic sensory neuropathy. $J$ Peripher Nerv Syst. 2003;8(4):227-235. doi:10.1111/j.1085-9489.2003.03028.x

42. Chowdhury SK, Dobrowsky RT, Fernyhough P. Nutrient excess and altered mitochondrial proteome and function contribute to neurodegeneration in diabetes. Mitochondrion. 2011;11(6):845-854. doi:10.1016/j.mito.2011.06.007

43. Morrison RS, Kinoshia Y, Johnson MD, et al. p53-dependent cell death signaling in neurons. Neurochem Res. 2003;28(1):15-27. doi:10.1023/a:1021687810103

44. Zhou H, Hu S, Jin Q, et al. Mff-dependent mitochondrial fission contributes to the pathogenesis of cardiac microvasculature ische$\mathrm{mia} /$ reperfusion injury via induction of mROS-mediated cardiolipin oxidation and HK2/VDAC1 disassociation-involved mPTP opening. $J$ Am Heart Assoc. 2017;6(3):e005328. doi:10.1161/ JAHA.116.005328

45. Zhou H, Du W, Li Y, et al. Effects of melatonin on fatty liver disease: the role of NR4A1/DNA-PKcs/p53 pathway, mitochondrial fission, and mitophagy. $J$ Pineal Res. 2018;64(1):e12450-e12452. doi:10.1111/jpi.12450 
46. Oren M. Decision making by p53: life, death and cancer. Cell Death Differ. 2003;10(4):431-442. doi:10.1038/sj.cdd.4401183

47. Rahimi A, Lee YY, Abdella H, et al. Role of p53 in cAMP/PKA pathway mediated apoptosis. Apoptosis. 2013;18(12):1492-1499. doi:10.1007/s10495-013-0895-6

48. Xiong H, Pang J, Yang H, et al. Activation of miR-34a/SIRT1/p53 signaling contributes to cochlear hair cell apoptosis: implications for age-related hearing loss. Neurobiol Aging. 2015;36(4):1692-1701. doi:10.1016/j.neurobiolaging.2014.12.034

49. Shi X, Pi L, Zhou S, et al. Activation of Sirtuin 1 attenuates high glucose-induced neuronal apoptosis by deacetylating p53. Front Endocrinol (Lausanne). 2018;9:274. doi:10.3389/fendo.2018.00274

50. Zhang L, Zou J, Chai E, Qi Y, Zhang Y. Alpha-lipoic acid attenuates cardiac hypertrophy via downregulation of PARP-2 and subsequent activation of SIRT-1. Eur $J$ Pharmacol. 2014;744:203-210. doi:10.1016/j.ejphar.2014.09.037

51. Hosseini A, Abdollahi M. Diabetic neuropathy and oxidative stress: therapeutic perspectives. Oxid Med Cell Longev. 2013;2013:168039. doi:10.1155/2013/168039

52. Nagamatsu M, Nickander KK, Schmelzer JD, et al. Lipoic acid improves nerve blood flow, reduces oxidative stress, and improves distal nerve conduction in experimental diabetic neuropathy. Diabetes Care. 1995;18(8):1160-1167. doi:10.2337/diacare.18.8.1160
53. Nickander K, McPhee B, Low P, Tritschler H. Alpha-lipoic acid: antioxidant potency against lipid peroxidation of neural tissues in vitro and implications for diabetic neuropathy. Free Radic Biol Med. 1996;21(5):631-639. doi:10.1016/0891-5849(96)00172-4

54. Stevens MJ, Obrosova I, Cao X, van Huysen C, Greene DA. Effects of DL-alpha-lipoic acid on peripheral nerve conduction, blood flow, energy metabolism, and oxidative stress in experimental diabetic neuropathy. Diabetes. 2000;49(6):1006-1015. doi:10.2337/ diabetes.49.6.1006

55. Cao J, Chen Z, Zhu Y, et al. Huangqi-Honghua combination and its main components ameliorate cerebral infarction with Qi deficiency and blood stasis syndrome by antioxidant action in rats. $J \quad$ Ethnopharmacol. 2014;155(2):1053-1060. doi:10.1016/j. jep.2014.05.061

56. Kim S, Kang IH, Nam JB, et al. Ameliorating the effect of astragaloside IV on learning and memory deficit after chronic cerebral hypoperfusion in rats. Molecules. 2015;20(2):1904-1921. doi:10.3390/ molecules20021904

57. Liu X, Zhang J, Wang S, Qiu J, Yu C. Astragaloside IV attenuates the $\mathrm{H}_{2} \mathrm{O}_{2}$-induced apoptosis of neuronal cells by inhibiting $\alpha$-synuclein expression via the p38 MAPK pathway. Int J Mol Med. 2017;40 (6):1772-1780. doi:10.3892/ijmm.2017.3157

\section{Publish your work in this journal}

Diabetes, Metabolic Syndrome and Obesity: Targets and Therapy is an international, peer-reviewed open-access journal committed to the rapid publication of the latest laboratory and clinical findings in the fields of diabetes, metabolic syndrome and obesity research. Original research, review, case reports, hypothesis formation, expert opinion and commentaries are all considered for publication. The manuscript management system is completely online and includes a very quick and fair peer-review system, which is all easy to use. Visit http://www.dovepress.com/testimonials.php to read real quotes from published authors. 